\title{
1 Data-driven train set crash dynamics simulation
}

2 Zhao Tang ${ }^{1}$, Yunrui Zhu ${ }^{1}$, Yinyu $\mathrm{Nie}^{1}$, Shihui $\mathrm{Guo}^{2}$, Fengjia Liu ${ }^{1}$, Jian

3 Chang $^{3}$, Jianjun Zhang ${ }^{1,3}$

41 State Key Laboratory of Traction Power, Southwest Jiaotong University, Chengdu, 5 China

62 School of Software, Xiamen University, Xiamen, China

73 National Centre for Computer Animation, Bournemouth University, Poole, UK 8

9 tangzhao@swjtu.edu.cn 10 


\section{Data-driven train set crash dynamics simulation}

Traditional finite element methods are arguably expensive in computation / simulation of the train crash. High computational cost limits their direct applications in investigating dynamic behaviours of an entire train set for crashworthiness design and structural optimisation. On the contrary, multi-body modelling is widely used because of its low computational cost with the great trade-off in accuracy. In this study, a data-driven train crash modelling method is proposed to improve the performance of a multi-body dynamics simulation of train set crash without increasing the computational burden. This is achieved by the parallel random forest algorithm, which is a machine learning approach that extracts useful patterns of force-displacement curves and predicts a force-displacement relation in a given collision condition from a collection of offline finite element simulation data on various collision conditions, namely different crash velocities in our analysis. Using the finite element simulation results as a benchmark, we compared our method with traditional multi-body modelling methods found that our data-driven method improves the accuracy over traditional multi-body models in train crash simulation and runs at the same level of efficiency.

Keywords: train sets crash, data-drive modelling, dynamics simulation, crash dynamics, parallel random forest, machine learning

\section{Introduction}

The crashworthiness of train structures plays a vital role in the performance of passive safety of train vehicles and determines the life and property safety of passengers $[1,2]$. Taking the vast economic expenditure, human labour and time costs of train crash experiments into considerations, a large amount of analyses of train crashworthiness are based on nonlinear finite element (FE) simulation [3].

In crashworthiness studies, especially those involving in iteration calculation and numerical optimisation processes, FE methods suffer from the high computation costs. It is common to use one or two vehicles to avoid the complexity of modelling a full train set to conduct these simulation cases [3]. 
2 efficiency and a tolerable computation accuracy [4]. MB modelling has been widely

3 adopted in multi-vehicle train crash simulation, this has an additional benefit of

4 removing the burden of FE methods in demanding for a well-built fine mesh model [5].

5 Lu [6] used lump mass models to simulate crashes between two train sets of a different

6 number of vehicles. It pointed out that the maximal crash force of the lead car is mainly

7 related to the first three vehicles and three or four vehicles are sufficient for investigat-

8 ing the dynamic behaviours of the train sets. Ambrósio and Dias [7, 8, 9] applied a non-

9 linear force element in a MB model to approximate the train's energy absorbing

10 components and carried out a train collision simulation with eight vehicles to optimise

11 their structural crashworthiness where lateral and vertical degrees of freedom were con-

12 sidered. Han [10] built a 3D train collision model based on MB methods to simulate the

13 train's behaviours in both head-on and oblique collision cases. Sun [11] used a three-

14 dimensional nonlinear MB model to simulate the collision behaviours of passenger ve-

15 hicles by taking the factors of wheel-rail contacts, coupler connections and the number

16 of vehicles into considerations. Zhou [12] made an investigation on a collision-induced

17 derailment train accident based on MB modelling.

18 Although the multi-body-based simulation can reasonably reflect the state of

19 motion in a train crash, there are series of undetermined model and material parameters

20 (e.g. Young's modulus, Poisson's ratio, etc.) in MB models and many of them still need

21 to be decided by more accurate methods. Many researchers extract these parameters

22 from experimental measurement or numerical simulation data, and some of them were

23 applied in automobile collision $[13,14]$ and vehicle dynamics $[15,16]$. By using the

24 lumped mass model, Pawlus [13] proposed a dynamics model for automobile collision

25 based on the real experiment data of typical automobile to pole collision. The accuracy 
1 of this model was verified by predicting the acceleration information under a new initial

2 velocity and comparing with the results from real experiments. Taheri [15] applied a

3 stochastic model based machine learning method to simulating the dynamic behaviour

4 of the second suspension of vehicle bogie. In the field of crashworthiness design of

5 trains, Wang and Zhou [16] simulated the nonlinear characteristics of vehicle's end

6 structures and couplers based on the force-displacement curve of energy absorbing

7 components, and analysed the dynamic behaviours in a head-on train crash scenario.

8 Baykasoglu [17] derived the stiffness-displacement curve in an equivalent spring-mass

9 model using the train crash simulation data under the initial velocity at $25 \mathrm{~m} / \mathrm{s}$, and ap-

10 plied this characteristic curve into the end structure of the train's MB model. Although

11 these MB-based modelling methods can extract model parameters from experiment or

12 simulation results to build a qualified crash simulation system, many of them only con-

13 sider a certain crash velocity. This limitation makes it difficult to generalise the findings

14 from a certain crash velocity to different crash velocities. Because the crash velocity has

15 a large influence on the parameters of the MB model, it is difficult to maintain an over-

16 all accuracy in simulating train crash cases at different initial velocities with the same

17 model parameters.

18 In this paper, we proposed a data-driven train crash modelling (DDTCM) meth-

19 od to improve the accuracy of traditional multi-body dynamics models in calculating

20 train set crash under different initial velocities. The framework of our method is pre-

21 sented in Figure 1. Firstly, FEM calculation results of train crash are collected to con-

22 struct a database of force-displacement curves. Second, based on the database of calcu-

23 lation results of single or multiple vehicle crash, we estimate the force-displacement

24 curve of the vehicle' end structure at a new initial velocity using a machine learning al-

25 gorithm. Third, a refined dynamics simulation results would be predicted by adopting 
1 these generalised curves into the corresponding MB model. Through validation, we

2 show in the latter part of this paper that our method can acquire satisfied simulation

3 results with only a small-scale database. Our main contributions are as follows:

4

- We explained that different initial velocities lead to different force-displacement curves in train collision by using FE simulation, which is contrary to the hypothesis that the initial velocity has little effect on the force-displacement curve of the train crash.

- We proposed a novel data-driven train crash modelling (DDTCM) to predict the dynamic information such as the acceleration and velocity, etc., and made a detailed comparison with other two existing MB simulation models. The results shown that our model presents a relatively high efficiency and accuracy.

- The Parallel Random Forest algorithm in the DDTCM shown a strong ability in predicting the force-displacement curve, which achieved a commendable accuracy with only a few training samples.

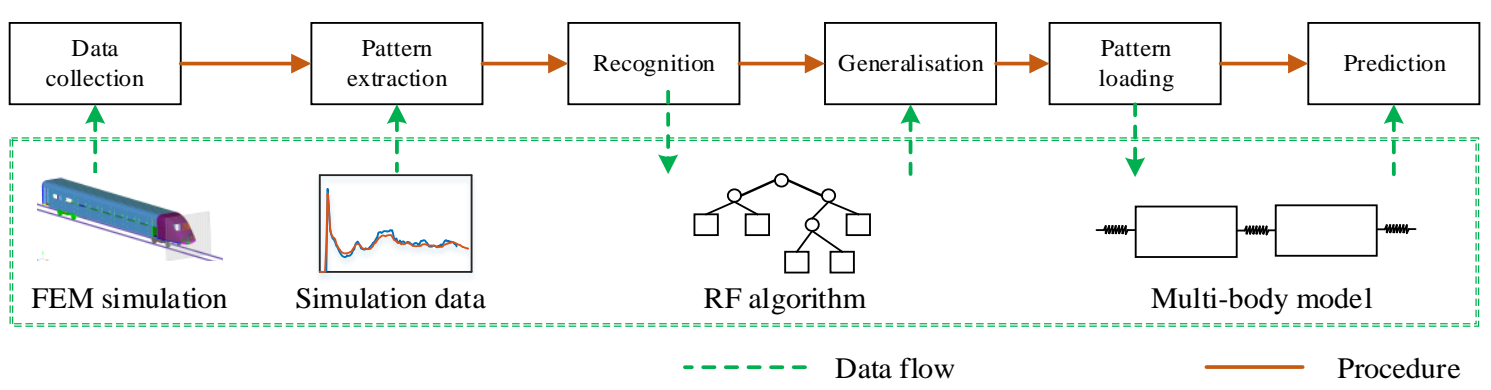

Figure 1 The framework and data flow of DDTCM

The rest of this paper is structured as follows. The data-driven train crash modelling (DDTCM) is presented in section 2. In section 3.1, the multi-body models are built at the last stage in DDTCM for predicting acceleration and velocity information. Sec- 
1 tion 3.2 mainly introduced two existing data-based simulation methods, which are

2 planned to make a comparison with our method in section 4. The conclusion and discus-

3 sions are presented in section 5.

\section{2. Data-driven train crash modelling (DDTCM)}

\section{2.1. Motivation}

6 In simulating the train crash under different initial velocities, the traditional MB model-

7 ling usually assumes the end structure's force-displacement relation as constant. How-

8 ever, our simulation results shown that this assumption is over-simplified and thus it is

9 inappropriate to generalise the force-displacement curve from a known fixed velocity

10 condition to those under different velocities. Figure 2 illustrates different deformation

11 profiles when a lead car crashed with a rigid wall at four different initial velocities. The

12 deformation pattern of each case is different from the others as shown in the region

13 highlighted in the yellow box. The corresponding force-displacement curves are shown

14 in Figure 3. In the initial stage of the train crash, the reaction forces of different initial

15 velocities are distinct after the lead car touched the rigid wall. Since the structure's

16 force-displacement relation is dependent on a specified initial velocity, predicting this

17 relation is important to understand the physical phenomena.

18 In Figure 2, the first and second crossbeam bend under $25 \mathrm{~km} / \mathrm{h}$, meanwhile the

19 structure near the second crossbeam undergoes a plastic deformation. The maximal

20 plastic strain value is 0.733 . In terms of the case under $36 \mathrm{~km} / \mathrm{h}$, there is an obvious bend

21 in the first crossbeam and the main plastic deformation part of this structure shows a

22 forward move. The maximal plastic strain value is 0.865 . In the case of $48 \mathrm{~km} / \mathrm{h}$, there

23 is no bend happening in the crossbeam. The deformation mainly occurs on the forepart

24 of the first crossbeam at the underframe of the lead car. Its maximal plastic strain value 
1 is 0.929 . In the case with initial velocity at $60 \mathrm{~km} / \mathrm{h}$, the plastic deformation at the fore-

2 part of the underframe is intensive with maximal plastic strain is 1.047 . Therefore, with

3 the increase of the initial impact velocity, the plastic deformation areas move forward

4 gradually, and the maximal plastic strain value shows an upward trend. In other words,

5 the corresponding impact force at the interface increases with the ascending of initial

6 velocity.

7

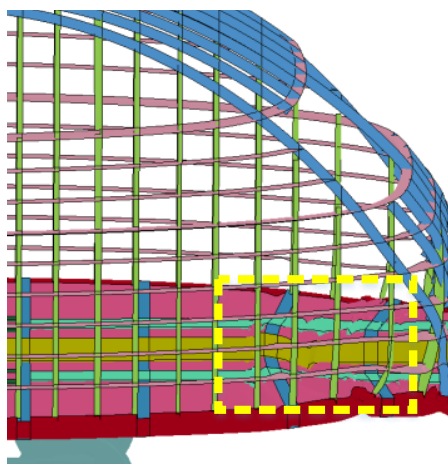

(a) $25 \mathrm{~km} / \mathrm{h}$

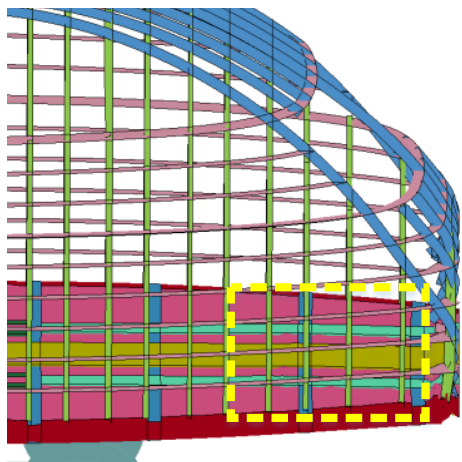

(c) $48 \mathrm{~km} / \mathrm{h}$

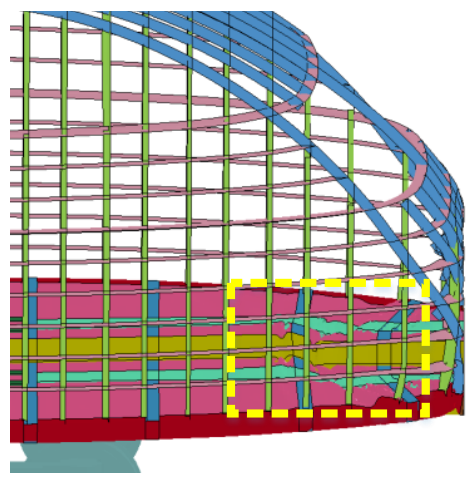

(b) $36 \mathrm{~km} / \mathrm{h}$

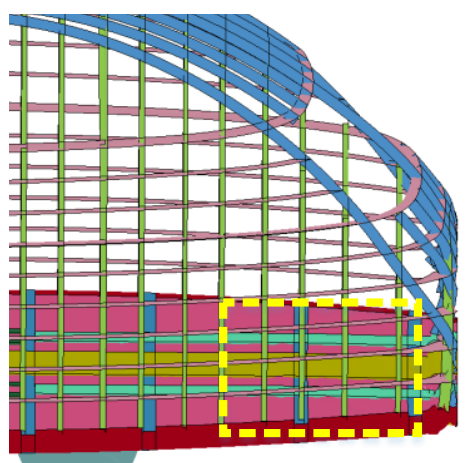

(d) $60 \mathrm{~km} / \mathrm{h}$

9 Figure 2 Deformation condition with different initial velocities in displacement at $10300 \mathrm{~mm}$

Hence in a certain range of initial velocity, the force-displacement curves are

13 similar, while if there is a large discrepancy in initial velocity values, their force-

14 displacement curves have considerable differences. Therefore, using one specific force-

15 displacement curve to calculate the deformation results with other initial velocities

16 would cause errors, which will also be validated in section 4. To address this concern, 
1 we used DDTCM method to recognise the relation between initial velocity and the cor-

2 responding force-displacement curve.

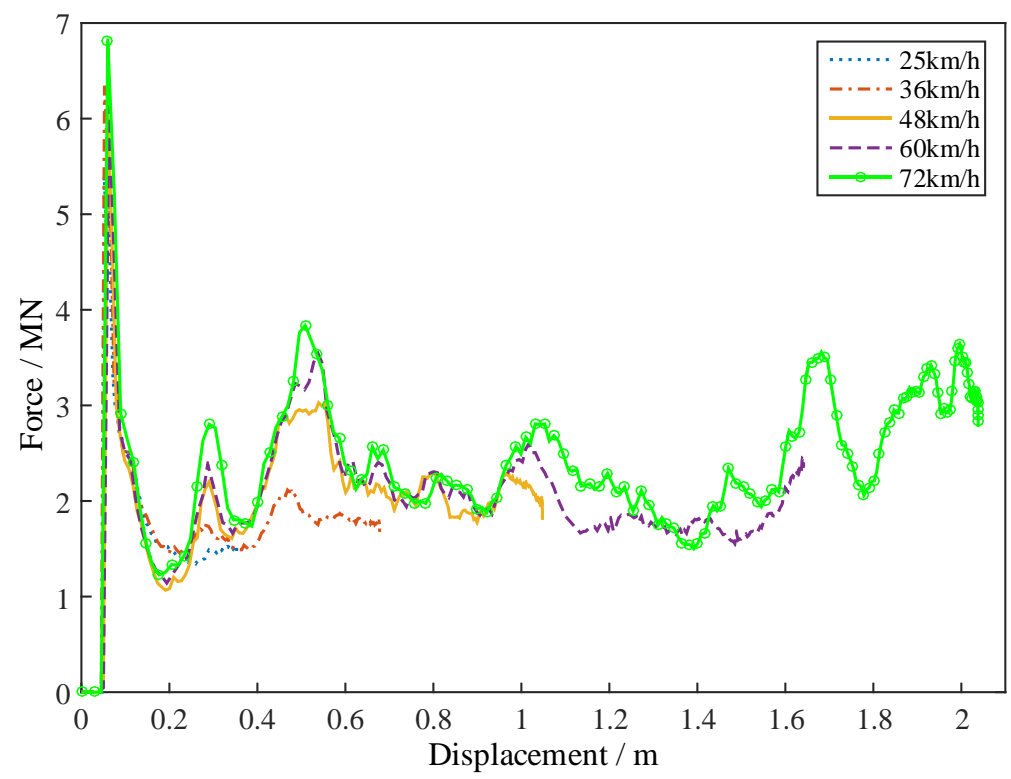

4 Figure 3 Five force-displacement curves under different initial crash velocities

5

\section{2.2. FE modelling of train crash}

7 To maintain the balance of accuracy and efficiency, we used the four-node shell ele-

8 ment to discrete the vehicle body. Considering that the main material of the vehicle

9 body is aluminium alloy, we adopted the bilinear isotropic and kinematic hardening ma-

10 terial model (MAT_3 in LS-DYNA) to express the elastic and plastic properties. The

11 FE model of the lead car and the vehicle are shown in Figure 4 (a) and (b). The main

12 parameters of the train model are presented in Table 1. More detailed FE modelling in-

13 formation can be found in our previous work [18].

14 


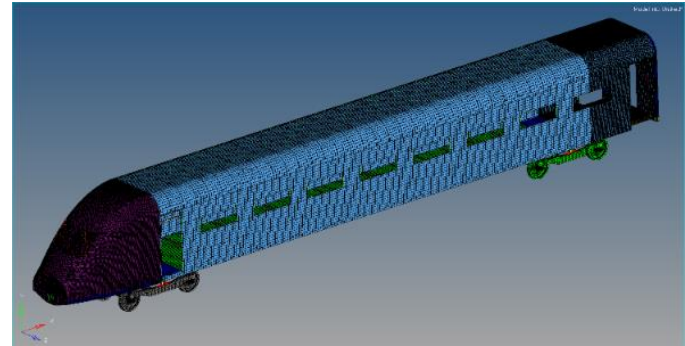

(a) FEM-model of the lead car

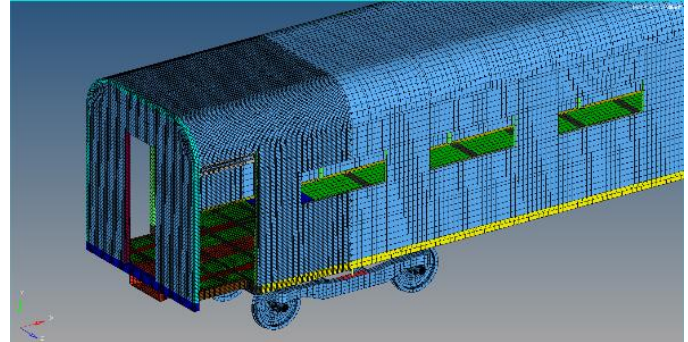

(b) FEM-model of the vehicle

3 Figure 4 FE models of the lead car and the passenger vehicle

4 Table 1. Parameters used in the vehicle FEM model

\begin{tabular}{cc}
\hline Parameter name & Value \\
\hline Mass of lead car $M_{h}$ & $24.00 \mathrm{ton}$ \\
Mass of vehicle $M_{v}$ & $20.24 \mathrm{ton}$ \\
Length of lead car $L_{h}$ & $27.60 \mathrm{~m}$ \\
Length of vehicle $L_{v}$ & $24.50 \mathrm{~m}$ \\
Length of driver's cab $L_{d}$ & $3.930 \mathrm{~m}$ \\
\hline
\end{tabular}

\subsection{Data preparation}

6 To take a full consideration of real train crash scenarios, we designed three different

7 simulation case groups, Group 1, Group 2 and Group 3, which are crash cases from lead

8 car to a rigid wall, lead car to lead car and vehicle to vehicle (see Figure 5). Different

9 initial velocities are applied to each train crash case. The grouping details and crash ve-

10 locity arrangements are presented in Table 2.

11 Table 2. Grouping details of different train crash scenarios

\begin{tabular}{ccc}
\hline Group No. & Crash scenario & Velocity $(\mathrm{km} / \mathrm{h})$ \\
\hline 1 & Lead car to rigid wall & \\
2 & Lead car to lead car & $25,36,48,60,72$ \\
3 & Vehicle to vehicle & \\
\hline
\end{tabular}

12

We limited the initial velocity below $160 \mathrm{~km} / \mathrm{h}$ in this paper. For a train, the ef-

14 fect of impacting a rigid wall (Group 1, Figure 5) with the initial velocity at $v$ is almost

15 equivalent to the effect of impacting a head-on train (Group 2, Figure 5) with the initial

16 velocity at $2 v$ [19]. Thus the real initial velocity range of Group 1 is practically from 50

17 to $144 \mathrm{~km} / \mathrm{h}$. The simulation results of Group 1 can be used to simulate the scenario of 
1 high-speed collision. The cases of Group 2 and Group 3 belong to the general cases

2 where the initial velocity ranges from 25 to $72 \mathrm{~km} / \mathrm{h}$. Since drivers are possible to trig-

3 ger an emergency brake before the collision happens, the initial velocity is less likely to

4 reach the maximal running velocity.

The Ls-Dyna is used to calculate all the simulation cases. The collision time is

$6300 \mathrm{~ms}$, and the history presents with 400 sample states (sampling period $d_{t}=0.75 \mathrm{~ms}$ ).

7 After the data are generated, they are fed into Parallel Random Forest (PRF) algorithm

8 in section 2.4 to predict an accurate force-displacement curve.

9

10

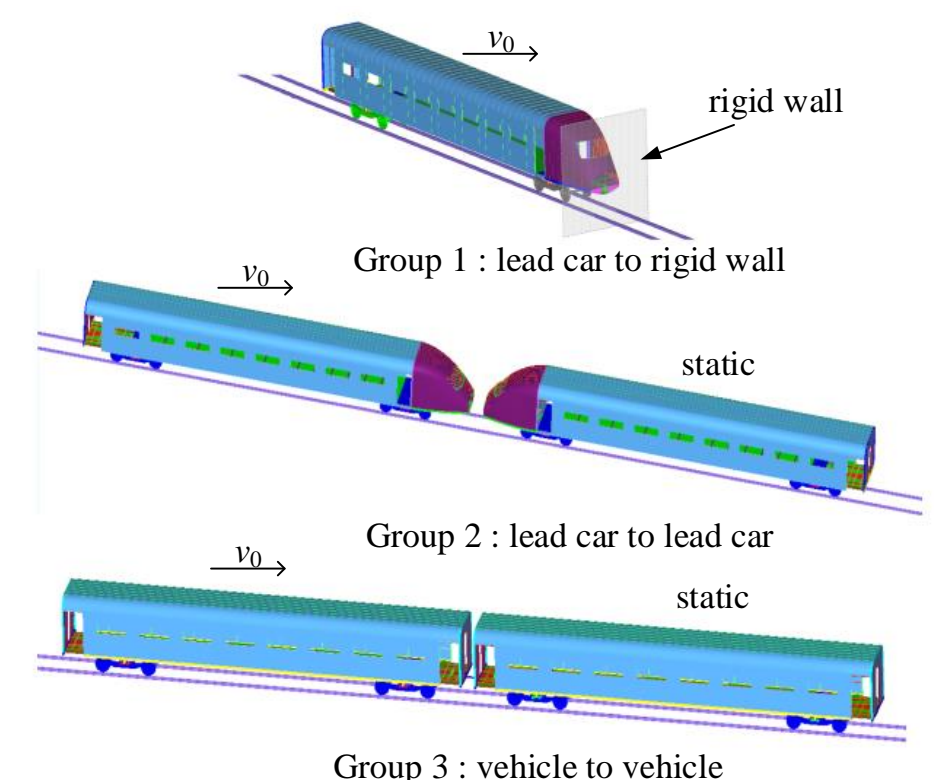

11 Figure 5 Illustration of three different train crash scenarios

\section{2.4. Random Forest prediction process}

14 Up to the present, in real-time simulation fields, the inverse modelling methods usually

15 use machine learning techniques to extract the latent mechanical patterns from existing

16 FE calculation results. These methods have a completed theory system in machine

17 learning fields, which is superior to traditional theory-oriented mechanical modelling 
1 (i.e. FEM) in calculation speed and modelling efficiency. Nevertheless, building a simu-

2 lation method completely based on machine learning methods may face several chal-

3 lenges in constructing hypotheses sets for the lack of specific knowledge about the

4 physical mechanism. Due to the fact that the multi-body dynamics models are able to

5 calculate all the fundamental dynamics information with high efficiency, we proposed a

6 data-driven train crash modelling method to refine the multi-body models with the help

7 of FE calculation results.

Parallel Random Forest (PRF) is an effective machine learning algorithm in

9 solving regression and classification problems [20, 21]. With the PRF algorithm, we can

10 use the existing force-displacement curves calculated by FEM to predict a new curve at

11 any initial velocity. Suppose that the database has $m$ FE calculation cases. The $k$ th case

12 is calculated based on the initial velocity at $v_{0}^{k}(k=1, \ldots, m)$. For the $k$ th result, the

13 force-deformation curve is extracted and expressed as $\left(d_{k}(t), f_{k}(t)\right)(t=1,2, \ldots, T)$

14 where $d_{k}(t)$ and $f_{k}(t)$ represent the displacement and force in the $t$ th second respec-

15 tively. $T$ is the maximal length of the sampling time. Then the force-displacement rela-

16 tion in the $k$ th result can be expressed by $F_{k}(\cdot)$

$$
f_{k}(t)=F_{k}\left[d_{k}(t)\right],(t=1,2, \ldots, T) .
$$

18 Denote the new velocity value by $v_{0}^{N}$, then the undetermined force-displacement rela19 tion in a new case can be presented as

$$
f^{N}(t)=F^{N}\left[d^{N}(t)\right]
$$

21 Where $d^{N}(t)$ and $f^{N}(t)$ represent the time series of the displacement and force respectively with given a new initial velocity $v_{0}^{N}$. 
The main objective of this paper is to generalise a new force-displacement rela-

2 tion $F^{N}$ based on the offline samples $\left\{v_{0}^{k}, F_{k}\right\}(k=1, \ldots, m)$. Based on comparisons, we

3 found out that the maximal displacement $\max _{t \in[1, T]}\left[d_{k}(t)\right]$ of train crash has the rela-

4 tion $v_{0}^{k} \propto \max _{\mathrm{t} \in[1, \mathrm{~T}]}\left[d_{k}(t)\right]$ for different values of the initial velocity $v_{0}^{k}$. In this case, a

5 cubic spline function is utilised to interpolate the scatter points

6 of $\left(v_{0}^{k}, \max _{\mathrm{t} \in[1, \mathrm{~T}]}\left[d_{k}(t)\right]\right), k=1,2, \ldots, m$, which produces a satisfactory prediction of

7 the $\max _{\mathrm{t} \in[1, \mathrm{~T}]}\left[d^{N}(t)\right]$ when given a new initial velocity $v_{0}^{N}$.

8 To acquire the $F^{N}$, we applied the parallel random forest algorithm as the data-

9 driven method. The inputs of the training set are defined as $\left\{v_{0}^{k}, d_{k}(t)\right\}$, and output

10 as $f_{k}(t)(k=1,2, \ldots, m)(t=1,2, \ldots, T)$. The first step of construct a random forest is

11 building a regression tree with non-prune [22].

12 All the training samples in a parent node are denoted by $\left\{v_{0}^{p}, d^{p} ; f^{p}\right\}$, and the coefficient of variation of the output by $C V^{p}$, where

$$
C V^{p}=\operatorname{Var}\left(f^{p}\right) / \operatorname{Mean}\left(f^{p}\right)
$$

15 Define the impurity function as

$$
I^{p}=\operatorname{Var}\left(f^{p}\right)
$$

17 The segmentation criterion of each parent node is finding $\operatorname{Input}_{i} \in\left\{v_{0}^{p}, d^{p}\right\}$ and $R_{i}$, which satisfy

$$
\arg \max _{\forall i, \text { Input }_{i}<R_{i}}\left[I^{p}-P^{l c} I^{l c}-P^{r c} I^{r c}\right]
$$

20 Where

$\left\{v_{0}^{l c}, d^{l c} ; f^{l c}\right\}=\left\{v_{0}^{p}, d^{p} ; f^{p} \mid\right.$ Input $\left._{i}<R_{i}\right\}$

$\left\{v_{0}^{r c}, d^{r c} ; f^{r c}\right\}=\left\{v_{0}^{p}, d^{p} ; f^{p} \mid\right.$ Input $\left._{i} \geq R_{i}\right\}$ 
$I^{l c}=\operatorname{Var}\left(f^{l c}\right), I^{r c}=\operatorname{Var}\left(f^{r c}\right)$,

$1 \quad P^{l}=\frac{N^{l c}}{N^{p}}, P^{r}=\frac{N^{r c}}{N^{p}}, N^{p}$ is the number of parent nodes. $N^{l c}$ and $N^{r c}$ are the number

2 of nodes in the two segments.

3 Each node is cut until its coefficient of variation is smaller than a threshold, then

4 the regression tree can be built.

The bootstrap method, a resampling method (detailed in [23]), is used here to

6 resample the training set to generate numerous different training subsets, and the regres-

7 sion tree is applied in each training subset. Afterwards, the numerous regression trees

8 are assembled to formulate the random forest [23]. Since there is no interference in each

9 couple of regression trees, an efficient parallel random forest algorithm can be obtained.

10 The framework of this algorithm is shown in Figure 6.

11

12

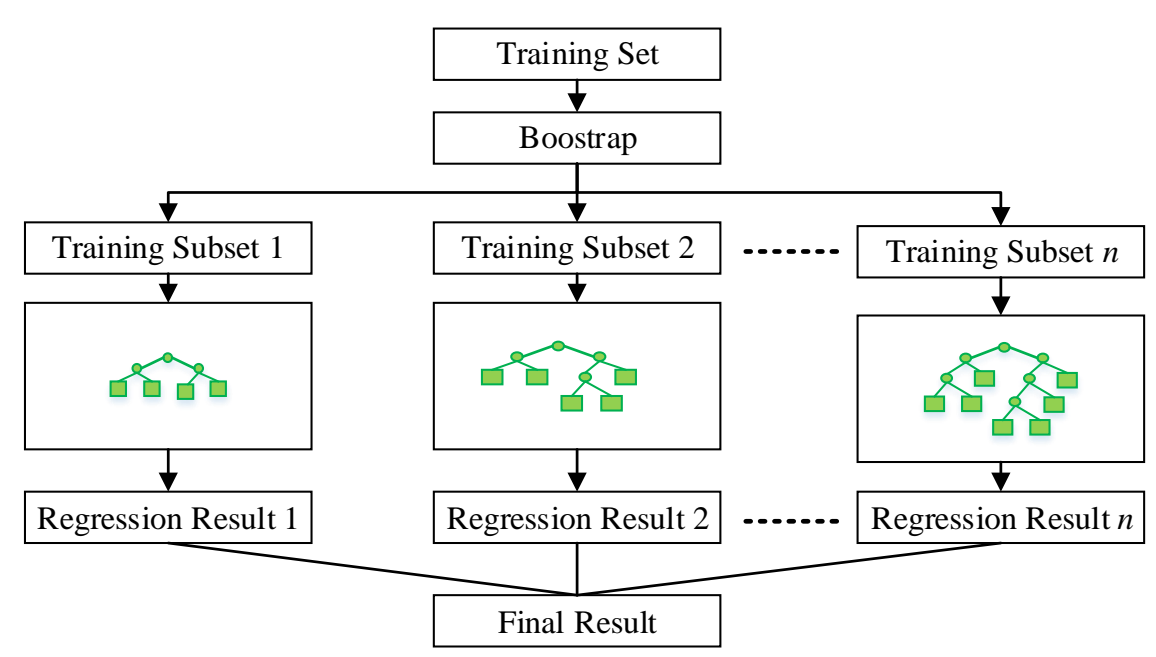

13 Figure 6 Framework of the parallel random forest regression algorithm

14

Since the $\max _{\mathrm{t} \in[1, \mathrm{~T}]}\left[d^{N}(t)\right]$ can be predicted via $v_{0}^{N}$, we can put new inputs

$$
\left[v_{0}^{N}, d^{N}(t) \mid d^{N}(t)<\max _{\mathrm{t} \in[1, \mathrm{~T}]}\left[d^{N}(t), t=1,2, \ldots, T\right]\right.
$$

17 to the random forest, so that the $f^{N}(t)(t=1,2, \ldots, T)$ can be predicted when averaging 
1 prediction values from all the regression trees. Applying the $\left[d^{N}(t), f^{N}(t)\right](t=$

$21,2, \ldots, T)$ as the force-displacement curve in the multi-body model constructed in sec-

3 tion 3.1, the acceleration and velocity information can be predicted accurately under the

$4 \quad$ initial velocity at $v_{0}^{N}$.

\section{3. Multi-body model construction for train crash simulation}

6 In low-speed crash scenarios, most of the kinetic energy is absorbed by couplers and 7 energy absorbing tubes, whereas, in high-speed case, deformable structures in train 8 body are also critical components to buffer enormous amount of energy. If the speed 9 reaches a high level, the vehicle would undergo a plastic deformation to absorb energy.

10 The train crash modelling can be classified by modelling the lead car, vehicle 11 and wheel-rail relation. More than $71 \%$ of train crash accidents belong to head-on or 12 rear-end collisions, hence it has important significance to build a longitudinal dynamics 13 model [24]. In longitudinal dynamics modelling, the wheel-rail relation can be neglect14 ed during the crash period [6].

\section{3.1. Longitudinal dynamics modelling of train sets}

16 Models used in vehicle crashworthiness research can be generally divided into damp-

17 ing-related models and non-damping models [13, 25]. The damping-related models con-

18 sider the damping effects as one part of the equivalent dynamics system, while non-

19 damping models use nonlinear spring elements to simulate the entire dynamic behav20 iours.

21 Damping effects exist in almost all mechanical systems. For train crash process, 22 the energy consumption is mainly comprised of deformation energy from structure de23 formation and thermal energy from friction, etc. and the mechanical energy from struc- 
1 ture deformation constitutes the main part of the total energy consumption. The core

2 idea of the damping-related model is using spring elements to simulate the nonlinear

3 vibration in collision and the damping effects to represent the energy consumption in

4 collision phase. Non-damping models are mainly constituted of nonlinear spring ele-

5 ments and mass blocks, which use reaction forces of springs to simulate the mechanical

6 characteristics in train crash.

$7 \quad$ The mass of the lead car is denoted by $m . k(x)$ and $c(\dot{x})$ indicate the spring's

8 stiffness curve and damper's damping curve respectively. Since the front and rear struc-

9 ture of the lead car are constructed by the driver's cab and the passenger vehicle respec-

10 tively, the spring and damper's characteristics of them are different, where we use

$11 k_{1}(x), k_{2}(x), c_{1}(\dot{x}), c_{2}(\dot{x})$ to distinguish.

12 Different from the damping relative models, the non-damping models simplify

13 the nonlinear characteristics in train crash process into a nonlinear spring. During the

14 collision phase, the spring is used as an energy storage component to store the system's

15 internal energy and potential energy. For the reason that there is no energy consuming

16 elements in non-damping models, all the mechanical energy and internal energy are

17 stored in the spring. In this study, the method for building an equivalent vehicle model

18 is similar to that for building a lead car model. The distinction between them is that the

19 spring and damper's characteristics of the front and rear structures in these vehicles are

20 identical, which means $k_{1}(x)=k_{2}(x), \quad c_{1}(\dot{x})=c_{2}(\dot{x})$.

\section{3.2. Traditional dynamics modelling methods}

22 There are two main simplified modelling methods in simulating vehicle crash. The first 23 one is nonlinear spring-damping model (NSDM) [11, 25] and the other one is force24 displacement relation model (FDRM) [12]. These two methods are usually used to pre- 
1 dict the dynamics information in vehicle crash process. In this section, we would build

2 these two models to make a comparison with our DDTCM method.

\section{3.2.1. Nonlinear spring-damping model (NSDM)}

4 NSDM is usually applied to approximate the acceleration and velocity information of 5 train crash using the calculation results by FEM, where piecewise functions are utilised

6 to express the characteristic curve of nonlinear spring and damper. The modelling 7 method proposed by Klausen [25] is widely used in vehicle crash scenarios, and we in-

8 troduce it to rail vehicle crash simulation in this paper. These undetermined parameters

9 in piecewise functions are treated as variables in this multi-body model. When given a 10 fixed initial velocity $v_{0}$, the acceleration, velocity and displacement of the train body 11 satisfy the following equation

$$
m \ddot{x}+c(\dot{x}, t) \dot{x}+k(x, t) x=0 .
$$

We set $\ddot{x}(t)$ as the acceleration at COG of the train body and $D\left(t, p^{m}\right)$ as the calculated acceleration curve from the multi-body model where $p^{m}$ is the undetermined parameters. The error function is set as

$$
e\left(t, p^{m}\right)=\left|D\left(t, p^{m}\right)-\ddot{x}(t)\right|^{T}\left|D\left(t, p^{m}\right)-\ddot{x}(t)\right| \text {. }
$$

These undermined parameters in NSDM are treated as variables in this error

18 function. The optimised $p^{m}$ will be obtained when minimising $e\left(t, p^{m}\right)$.

\subsubsection{Force-displacement relation model (FDRM)}

20 In order to simulate the dynamic process between two vehicles with convenience, Zhou

21 [16] derived the force-displacement curve of vehicle's energy absorbing components

22 with nonlinear FE method, and use this curve to simulate the characteristics of nonlinear 
1 spring between couplers. We call this model force-displacement relation model

2 (FDRM) in this paper. To refine its accuracy, we proposed the DDTCM that has a better

3 accuracy and generalisation ability. The main idea of the FDRM is using the crash cal-

4 culation results from FE data and extracting a force-displacement curve as the charac-

5 teristic curve of an equivalent spring, then applying this curve to a spring-mass model to

6 finish building the train crash model. The mechanical models of this method are illus-

7 trated in Figure 1. Five types of force-displacement curves with different initial velocity

8 based on Table 2, Group 1 are represented in Figure 3. It is apparent that there is a simi-

9 lar deformation pattern despite the difference of the initial velocity.

\section{4. Results and discussions}

11 In train crash dynamics study, the lead car is one of the most significant research objec-

12 tives [3]. So we take the crash scenario between the lead car and rigid wall for instance

13 to validate the performance of NSDM and FDRM methods. We solved all of them on

14 the Windows platform with Core i7, 3.40 GHz processor and 8GB RAM.

\section{4.1. Model validation for lead car crash scenarios}

\section{4.1.1. The performance of NSDM}

17 On account of the high structure complexity and nonlinear characteristics of the lead car,

18 we set more than five pieces for the piecewise function to express the nonlinear charac-

19 teristics in spring and damper. We tried 30 types of conditions and found that when the

20 characteristic curve of nonlinear spring and damper are set as 10 pieces and 5 pieces

21 functions respectively, the genetic algorithm can reach the relative lower error. Though

22 a more complex piecewise function can express the nonlinear relations more accurately,

23 it needs more time to optimise a large number of variables, which increases the likeli- 
1 hood of falling into a local minimum.

2 The predicted acceleration and velocity are shown in Figure 7 after optimised

3 model parameters are applied to the corresponding NSDM. The NSDM method has a

4 great performance in representing the fluctuation of acceleration curve and can tolerate

5 the local noise entwined with the overall trend, which is acceptable in engineering simu-

6 lation. On account of the great results in fitting acceleration curve, the predicted veloci-

7 ty nearly coincides with the calculated velocity via FEM.

Figure 8 displays the predicted characteristic curve of nonlinear spring and

9 damper, which illustrates that the stiffness of the spring is relatively large (at $7.58 \times 10^{7}$

$10 \mathrm{~N} / \mathrm{m}$ ) at the initial stage of the collision. After that, the stiffness curve shows a dramati-

11 cally downward trend and remains stable at about $1 \times 10^{6} \mathrm{~N} / \mathrm{m}$. The damping value also

12 increases remarkably at the initial stage of collision and reaches the value at $1.65 \times 10^{5}$

$13 \mathrm{~N} \cdot \mathrm{s} / \mathrm{m}$ at $17.3 \mathrm{~m} / \mathrm{s}$ and $13.9 \mathrm{~m} / \mathrm{s}$.

14

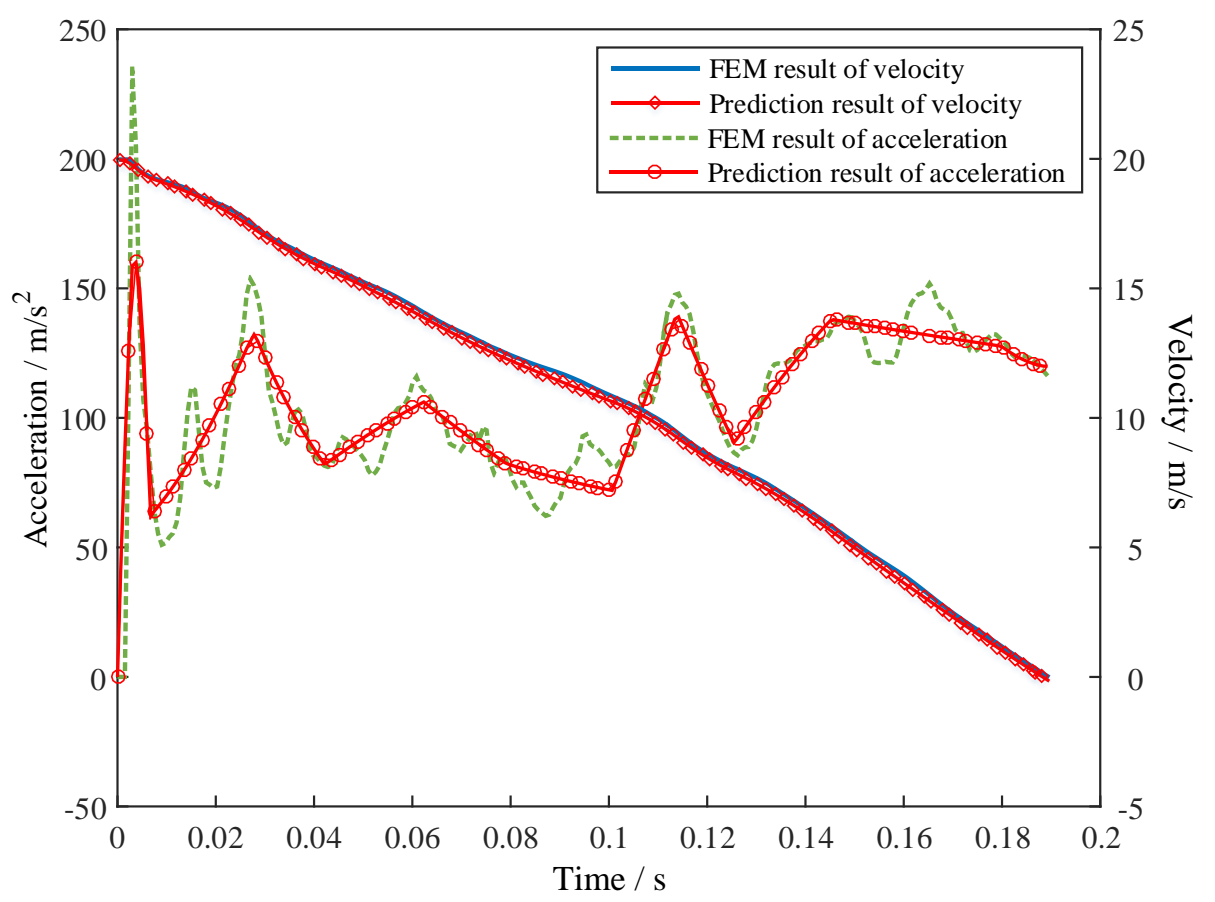

16 Figure 7 Predicted acceleration and velocity with optimised model parameters applied

17 to the corresponding NSDM 


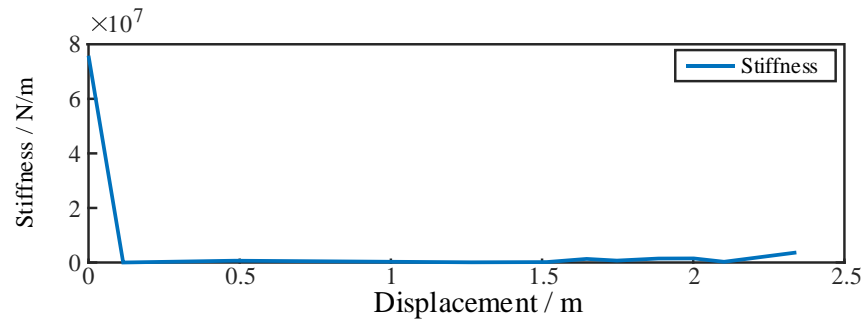

(a) Predicted result of stiffness

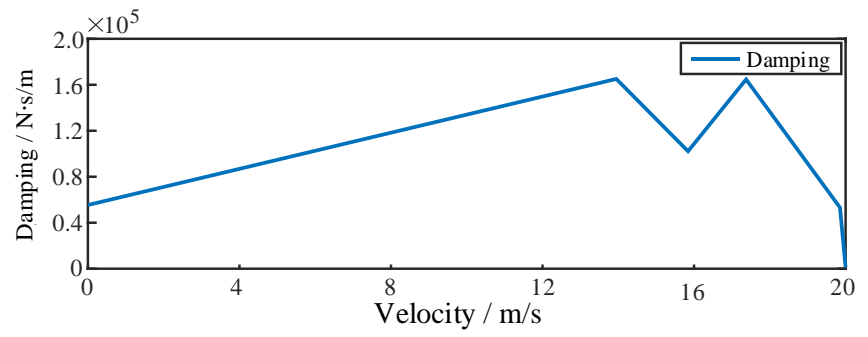

(b) Predicted result of damping

3 Figure 8 Predicted characteristics curves of the spring (a) and damper (b) based on 4 NSDM

5

\section{4.1.2. The performance of FDRM}

7 According to Group 1, Table 2, we use the crash calculation results based on the initial 8 velocities at 25, 36, 48, 60 and $72 \mathrm{~km} / \mathrm{h}$ from the FE method in Ls-Dyna, and extract the

9 force-displacement curve for each case, then apply these curves to the non-damping 10 model to pre-validate the feasibility of FDRM. The calculated velocity curves and ac11 celeration curves of the FE method and FDRM are shown in Figure 9.

12 From Figure 9, the pre-validation results show that by extracting force13 displacement curves from FE calculation results and applying them to a non-damping 14 model, the velocity and acceleration curves can be accurately reconstructed. However, a 15 concern is that the force-displacement curve of specified structure is fixed, while based 16 on our observation, these deformation pattern changes after the initial velocity is altered.

17 To accurately predict force-displacement curve at a new initial velocity, we use the 
1 DDTCM method to improve the FDRM and predict the force-displacement based on the

2 feasibility validation.

3

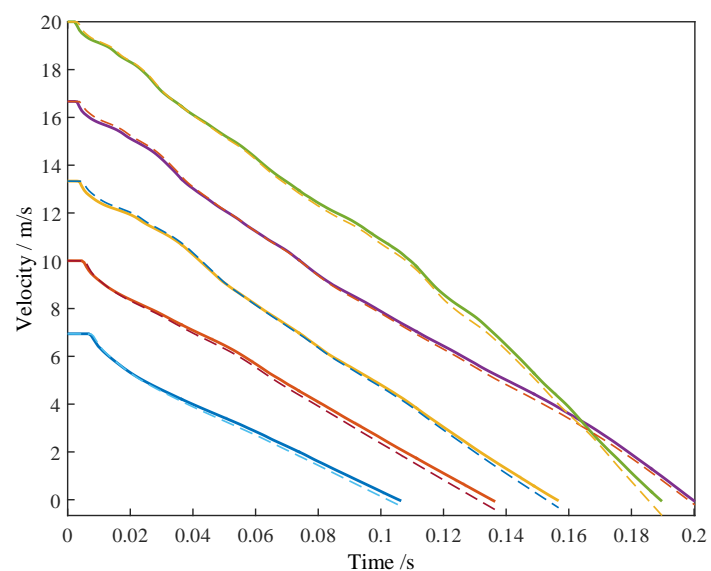

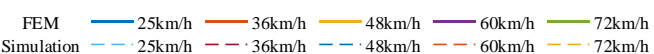

(a) Velocity

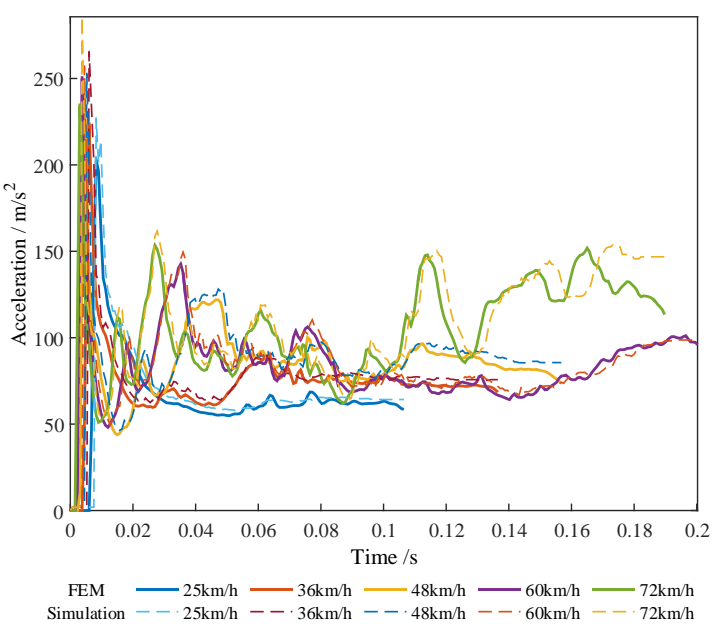

(b) Acceleration

\subsubsection{The performance of DDTCM with single vehicle}

11 When the initial velocity increases, the maximal displacement of crash generally shows 12 an upward trend. Therefore, the deformation curves under the initial velocity at $72 \mathrm{~km} / \mathrm{h}$

13 should be selected in the training set. In this case, we firstly use three deformation curves under the initial velocity at $25,48,72 \mathrm{~km} / \mathrm{h}$, and treat the curve under $48 \mathrm{~km} / \mathrm{h}$ as the test set while the others as the training set. Then the force-displacement curve under $48 \mathrm{~km} / \mathrm{h}$ can be predicted with 50 decision trees in the parallel random forest algorithm,

17 and the comparison with the corresponding FE calculation results is shown in Figure 10 
$2 \mathrm{~m}$, and the others are generally lower than $1 \mathrm{MN}$. The relative errors at the first two 3 peak points are $18.9 \%$ and $7.8 \%$ respectively, which shows a great performance in accu4 racy. If we use the four deformation curves under the initial velocity at $25,36,60,72$ $5 \mathrm{~km} / \mathrm{h}$ as training samples, a better prediction result can be obtained (Figure $10(\mathrm{~b})$ ).

6

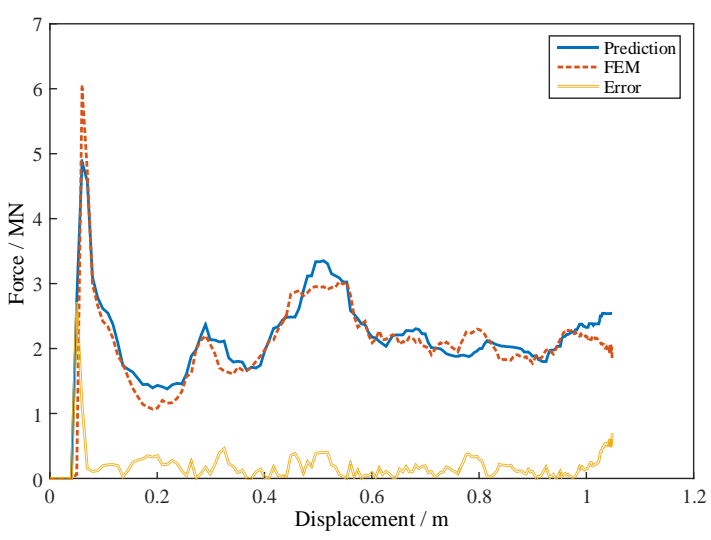

(a) Prediction result with 2 samples

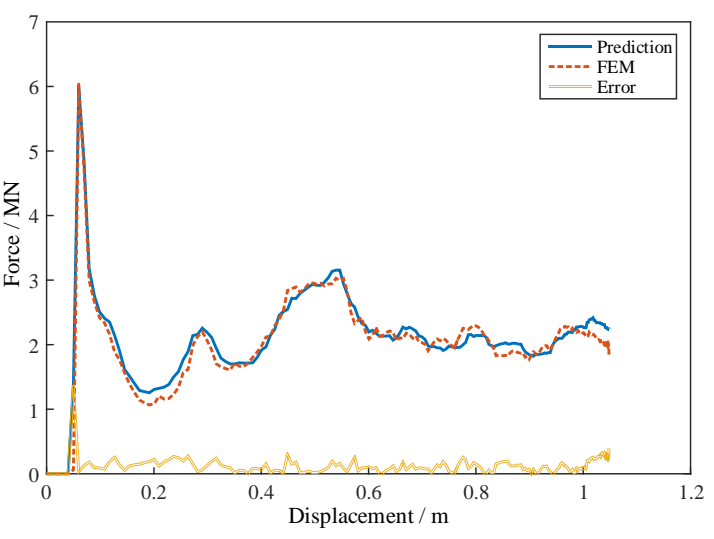

(b) Prediction result with 4 samples

Figure 10 Predicted force-displacement curves by DDTCM using two and four training samples

From Figure $10(\mathrm{~b})$, the maximal error of this predicted curve is $1.36 \mathrm{MN}$ at 0.05 $\mathrm{m}$, and the others are generally lower than $0.36 \mathrm{MN}$. The relative errors at the first two peak points are $1.2 \%$ and $4.5 \%$ respectively. That means, by using DDTCM, the prediction accuracy of the force-displacement curve is improved with an increasing number of training samples.

If we only adopt the force-displacement curve at the initial velocity of $72 \mathrm{~km} / \mathrm{h}$ as the training sample, this prediction method is reduced to FDRM. In view of this situation, we directly apply the force-displacement curve at the initial velocity at $72 \mathrm{~km} / \mathrm{h}$ to generalise that under the initial velocity at $48 \mathrm{~km} / \mathrm{h}$. The comparison result will be given in section 4.2 . 
2 In general conditions, a crash between two train sets of three or four vehicles are ade-

3 quate to represent the dynamic behaviours of longer trains [6]. To validate the perfor-

4 mance of DDTCM in simulating the train crash between two multi-vehicle train sets, a

5 crash simulation between two four-vehicle train sets was conducted by comparing with

6 the FEM result. Eight vehicles are involved in this case. According to the European

7 train crash standard EN15227:2008 [26], the initial velocity of passenger train's head-

8 on crash with other same-scale trains should be set at $36 \mathrm{~km} / \mathrm{h}$. The four deformation

9 curves under the initial velocity at $25,48,60,72 \mathrm{~km} / \mathrm{h}$ were used as training samples to

10 predict force-displacement curve at $36 \mathrm{~km} / \mathrm{h}$ with DDTCM method. The crash case in-

11 volving two train sets of four vehicles can be simulated using this predicted curve (see

12 Figure 11). The comparison results of acceleration and velocity of the first four vehicles

13 are shown in Figure 12 (a) and Figure 12 (b), and the results of the last four vehicles are

14 nearly symmetrical with those of the first ones.

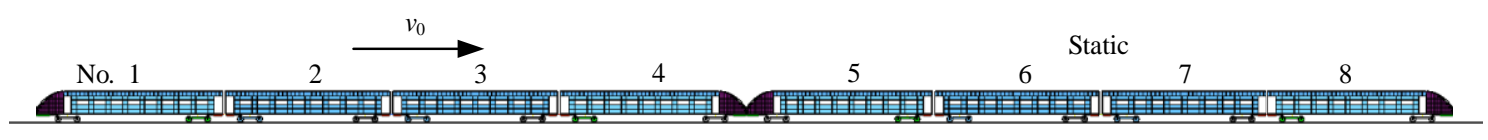

Figure 11 Head-on crash simulation between two 4-vehicle train sets

From Figure 12 (a), the velocity of the end structure of the lead car shows a de-

20 crease first at the impact velocity at $36 \mathrm{~km} / \mathrm{h}$, and the following vehicles start to slow

21 down successively afterwards. When the crash time arrives at $0.3 \mathrm{~s}$, these four vehicles

22 reach the same velocity. The DDTCM shows a great performance in fitting the velocity curves of these four vehicles in Figure 12 (a), where the maximal absolute error com- 
1 pared with FEM is $0.48 \mathrm{~m} / \mathrm{s}$ happened at $0.224 \mathrm{~s}$ in the No.3 vehicle. The overall rela-

2 tive error of the DDTCM is $2.34 \%$ which is an acceptable result.

3

4

5

6 Figure 12 Comparison result of the velocity and accelaration curves of the first vehicles

7 between using the FE method and DDTCM

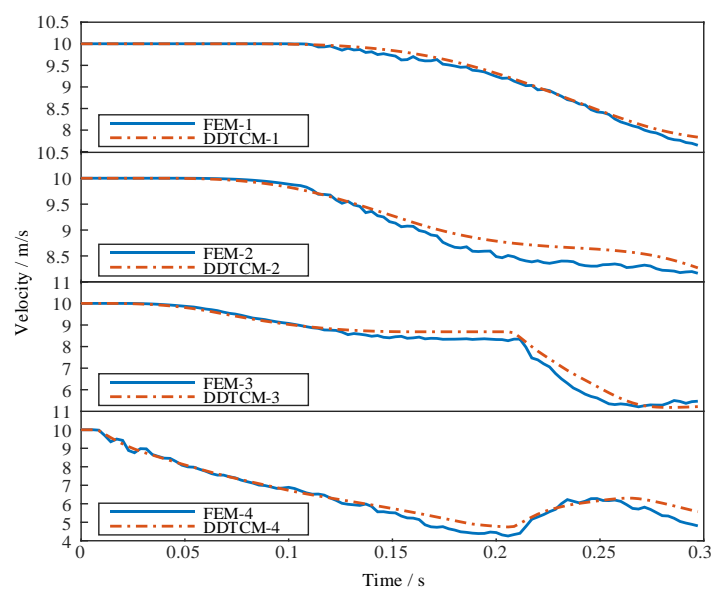

(a) Velocity

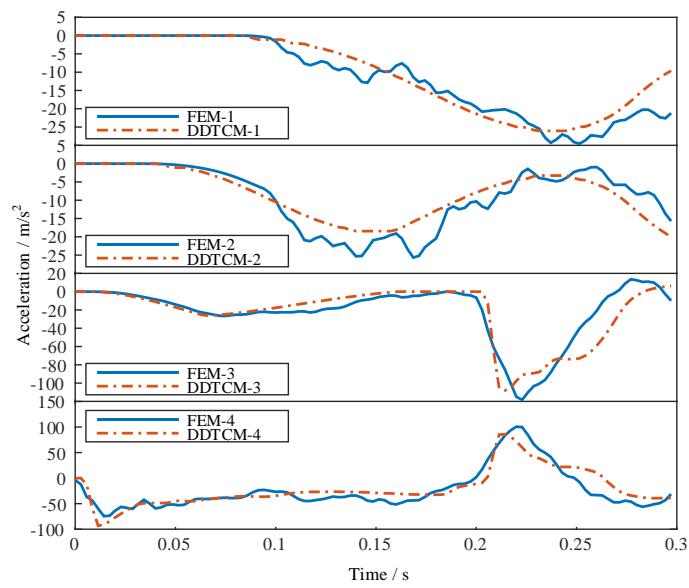

(b) Accleration

8

For the interaction between vehicles and couplers, the acceleration curves of

10 these vehicles show a drastic fluctuation during the crash (see Figure 12 (b)). For the

11 No.1, No.3 and No.4 vehicles, the overall trends of the predicted acceleration curves are

12 consistent with those of using the FE method except that there are phase differences of

13 acceleration peaks in No.3 vehicles. For the No.2 vehicle, the DDTCM method shows a

14 pleasing performance.

\subsection{Comparison}

16 This section presents the comparison of the results from three modelling methods:

17 NSDM, FDRM and DDTCM. 
2 the corresponding multi-body models to generate acceleration and velocity curves under

3 the initial velocity at $48 \mathrm{~km} / \mathrm{h}$. The comparison results are shown in Figure 13. sults via DDTCM are more approximate to that calculated via FEM both for accelera-

6 tion and velocity. As for acceleration prediction, before $0.13 \mathrm{~s}$, the accuracy of DDTCM

7 outperforms the other two methods. After $0.13 \mathrm{~s}$, the FDRM shows a slight superiority.

8 The NSDM method only shows a tolerable accuracy in fitting acceleration before 0.1

9 seconds. After that, it displays a descending accuracy.

10 For practical applications, the forepart of train crash period has the most inten-

11 sive impact which is the most important part for vehicle crashworthiness performance 12 and passenger's security protection. Therefore, we can see that the DDTCM has the best 13 accuracy in simulating the dynamics behaviours of the train crash. Because the predic14 tion part of DDTCM is accomplished by the multi-body model which is the same as 15 FDRM and NSDM, hence the calculation time efficiency is similar.

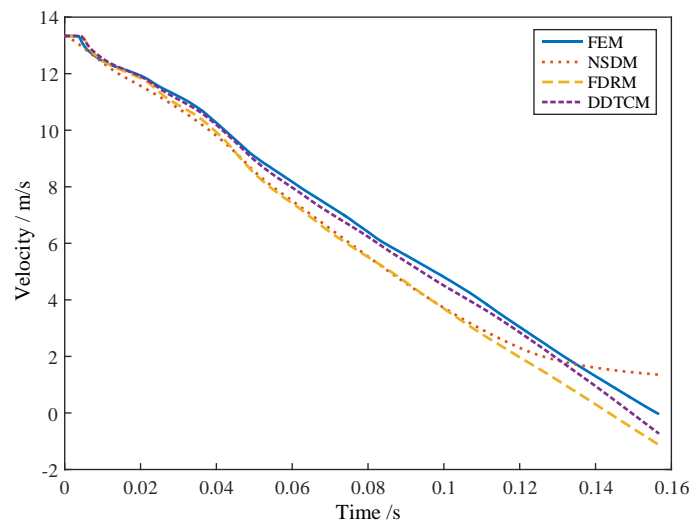

(a) Velocity

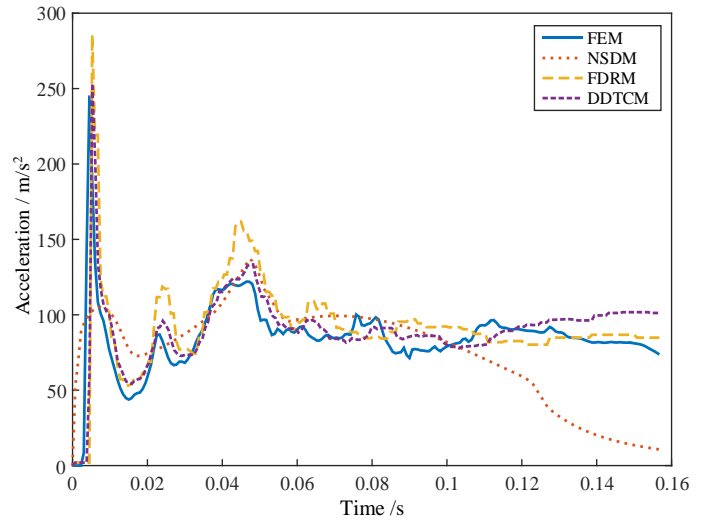

(b) Acceleration

19 Figure 13 Comparison of generalisation ability of acceleration and velocity between NSDM, FDRM and DDTCM 
2 above are shown in Table 3, where the FE calculation results are regarded as the

3 benchmark for the rest three methods.

4 Table 3. Computational efficiency and residual of 4 simulation methods

\begin{tabular}{cccccc}
\hline Method's name & VAveRE & AAveRE & Training time (mins) & $\begin{array}{c}\text { Prediction time } \\
\text { (mins) }\end{array}$ & $\begin{array}{c}\text { Simulation time } \\
\text { (mins) }\end{array}$ \\
\hline FEM & - & - & - & - & 355.61 \\
NSDM & $11.3 \%$ & $35.9 \%$ & - & 605 & $3.51 \times 10^{-4}$ \\
FDRM & $14.8 \%$ & $14.7 \%$ & - & - & $3.51 \times 10^{-4}$ \\
DDTCM & $4.10 \%$ & $11.7 \%$ & 1.53 & 0.003 & $3.51 \times 10^{-4}$ \\
\hline
\end{tabular}

5 Note: VAveRE is the average relative error of the predicted velocity; AAveRE is the average

6 relative error of the predicted acceleration.

From this table, these three methods can approximate the FE method with a tol-

9 erable accuracy in predicting the velocity curves. The maximal absolute and relative

10 errors of predicting velocity curves with DDTCM are about $0.744 \mathrm{~m} / \mathrm{s}$ and $4.10 \%$ re-

11 spectively. In predicting the acceleration curves, these three methods present errors in

12 different levels. The average error reaches $35.9 \%$. In Figure 7, we can see an acceptable

13 accuracy with NSDM in predicting the acceleration curve at the crash velocity at 36

$14 \mathrm{~km} / \mathrm{h}$, while it presents a large error when predicting those at the velocity of $48 \mathrm{~km} / \mathrm{s}$.

15 This explains that NSDM shows a low performance in generalisation ability. DDTCM

16 displays the highest accuracy in predicting acceleration among these methods. The rela-

17 tive error is $11.7 \%$. From the calculation results in Figure 13 (b), we can see phase lags

18 of the predicted curves by the FDRM and DDTCM comparing with those by the FEM.

19 These phase differences cause the fact that the acceleration peak calculated by FDRM

20 and DDTCM appear at a different time compared with that of using the FE method and

21 these lead to a large error in predicting acceleration curves. The NSDM method provid-

22 ed an excellent accuracy before $0.1 \mathrm{~s}$ and maintained a similar fluctuation pattern with 
1 the FE methods. In summary, DDTCM can present a great performance compared with

2 other methods no matter measured by maximal absolute error or by average relative er3 ror.

Besides that, DDTCM shows its advantage in time efficiency compared with the

$5 \quad$ FE method on the platform mentioned at section 4.1.1. In the case of Group 1 in Figure

6 5, the FE method costs about 355.61 mins, 232 times of that with using DDTCM.

\section{4.3. Application of DDTCM to train sets crash simulation}

8 In order to further evaluate the feasibility of DDTCM in train set crash simulation, we

9 built dynamics models for the lead car and vehicles respectively, and connect these

10 models end to end to construct the dynamics model for a train set (see Figure 14) which

11 can be used in train set crashworthiness research. The left 8-vehicle train set is at the speed of $v_{0}$ crashing with the right 8 -vehicle static train set. The details of the simulation 13 process and result are discussed in section 4.3.
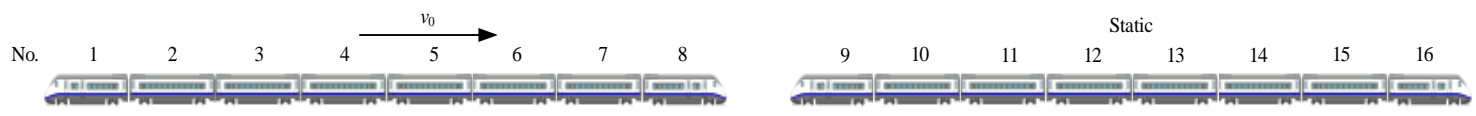

Figure 14 Head-on crash simulation between two 8-vehicle train sets

19 The initial velocity of the train set collision is $36 \mathrm{~km} / \mathrm{h}$. Each vehicle is set as a rigid

20 body whose end structures and couplers are substituted by nonlinear spring elements.

21 Based on FE calculation results in Group 2 and Group 3, Table 2, we use DDTCM to predict the force-displacement curve of each spring elements at the initial velocity of 36

$23 \mathrm{~km} / \mathrm{h}$ and apply these force-displacement curves in the corresponding spring elements in

24 train set's multi-body model to predict the velocity and acceleration of each vehicle 
From Figure 15 (a), the velocity of the active train shows a downward trend dur-

3 ing the process of the crash, meanwhile the static train begins speeding up. At the time

4 of $0.08 \mathrm{~s}$, the two vehicles reach the same velocity. From this phenomenon, the veloci-

5 ties of the middle vehicles in the two train sets obviously displays two stages, which

6 corresponds to different velocities when exerting force from couplers and end structures

7 respectively. After the velocity of each vehicle converges to a similar level, the minor

8 fluctuation of velocity shows that there is a secondary collision happens between vehi-

9 cles.

10

11

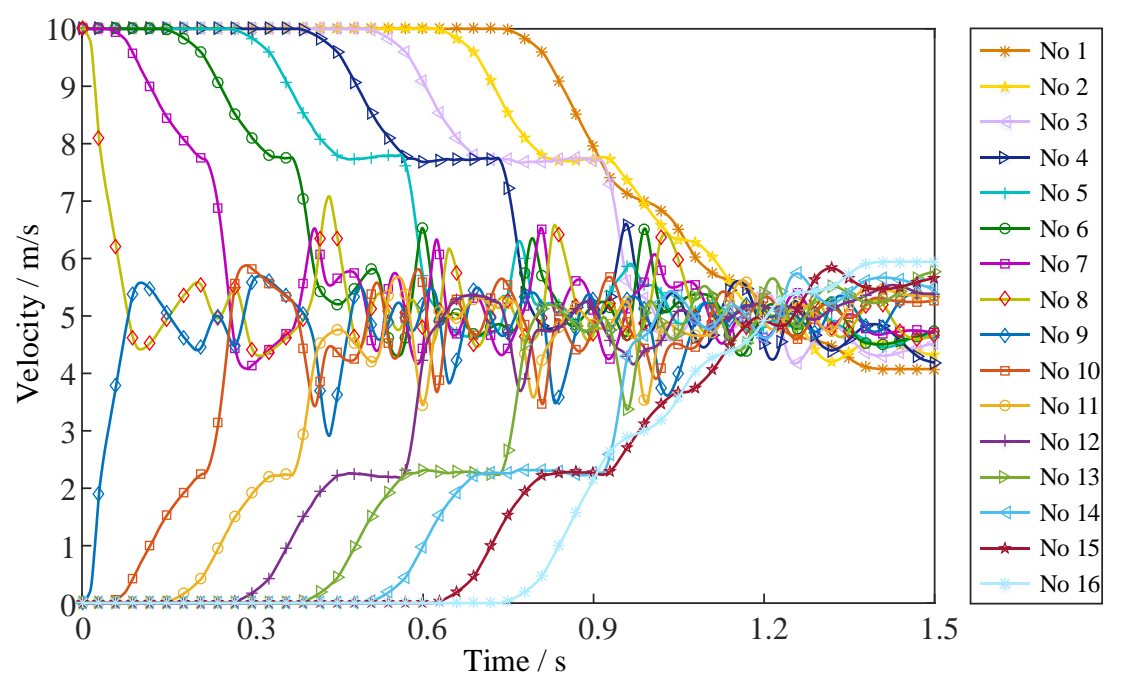

12

13

(a) Velocity

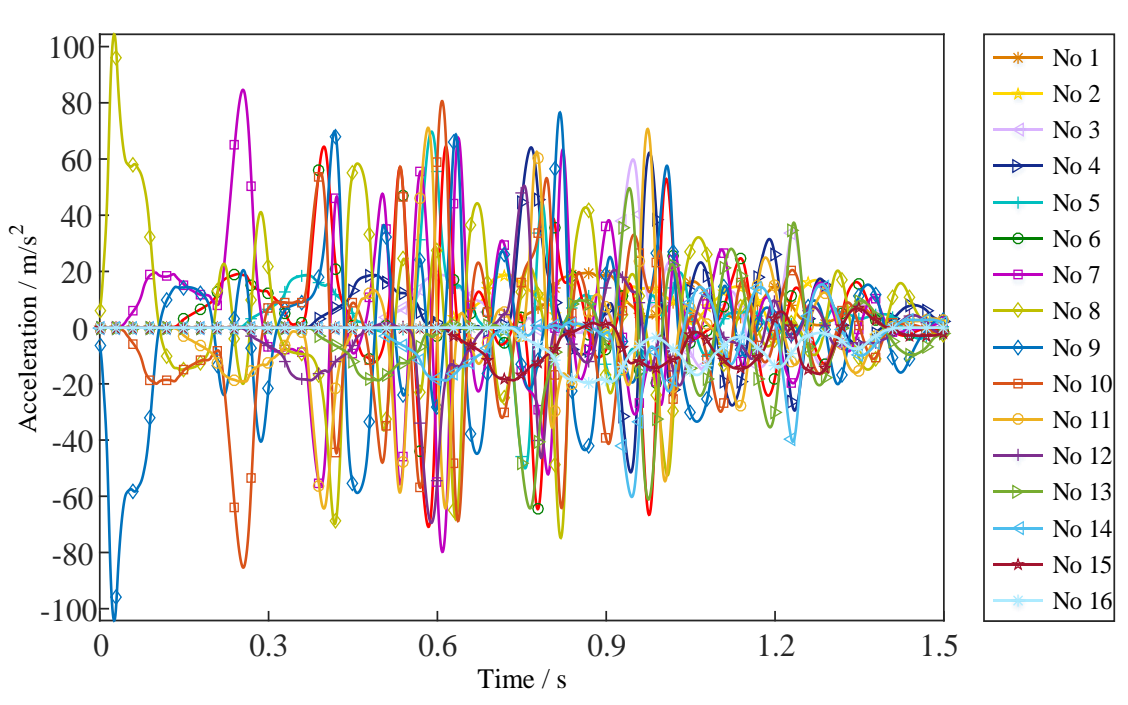


2 Figure 15 Velocity and acceleration prediction of each vehicle in a head-on crash simu3 lation between two 8-vehicle train sets.

From Figure 15 (b), the acceleration curves have multiple peak points, which

5 shows that the acceleration decreases with some time-varying fluctuations rather than a

6 strictly monotonic decrease. This is mainly because each vehicle in the two train sets

7 has a collision with the neighbouring vehicle. Since the two lead cars crash with each

8 other with the initial velocity, the first peak value of acceleration is maximal. When the

9 crash continues, the acceleration shows a fluctuated trend and gradually turns to be flat.

\section{5. Conclusions}

11 We proposed a data-driven method (DDTCM) to model the train set crash dynamics. Its 12 application to longitudinal dynamics modelling of train sets crash was discussed in de13 tail.

14 Existing methods in multi-body simulation generally ignore the issue of how the 15 initial velocity would affect the simulation process, particularly the force-displacement 16 curves. However, our validation experiments show that the initial velocity plays a criti17 cal role in determining the shape of the force-displacement curve. Therefore it is insuf18 ficient to use a force-displacement curve at a fixed velocity and generalise to the simu19 lation scenarios at other velocities.

20 The DDTCM model addresses the challenge by accurately reconstructing the 21 force-displacement curve at different initial velocities. To accomplish this goal, the Par22 allel Random Forest algorithm, which is a machine learning method, was employed to 23 predict the force-displacement curve from the offline FE simulation results under differ24 ent conditions and search for the optimal candidate for online MB simulation. The re- 
1 sults show that this strategy improves the accuracy of the simulation results of MB

2 models, without sacrificing its advantages in computational efficiency.

This study not only clarifies some critical issues which are generally ignored in

4 previous practices, but also offers a practical solution to engineers. The precondition of

5 using the DDTCM is that there is a similarity between force-displacement curves under

6 different initial velocities. That is to say the force-displacement curve should changes

7 continuously with the change of the initial velocity. Fortunately, structures like the ve-

8 hicles meet this precondition.

There are several interesting directions that are worth exploring in the future.

10 For example, at the current stage of this study, we only apply DDTCM to the longitudi-

11 nal dynamics modelling of train set crash which is a $1 \mathrm{D}$ model. How to extend it to 3D

12 train set crash modelling needs to be explored in the future.

Acknowledgements

15 This research was supported and funded by National Natural Science Foundation of 16 China (51405402, 51475394), the Self-Developed Research Project of the State Key

17 Laboratory of Traction Power (2015TPL_T06) and the Fundament Research Funds for 18 the Central Universities for the Central Universities (2682016CX128).

21 [1] Chadwick SG, Zhou N, Saat MR. Highway-rail grade crossing safety challenges 22 for shared operations of high-speed passenger and heavy freight rail in the US. Saf Sci. 23 2014;68:128-137.

24 [2] Kimura S, Mochida T, Kawasaki T, Nakamura H, Yamaguchi T. Evaluation of 25 Energy Absorption of Crashworthy Structure for Railway's Rolling Stock (Numerical 26 Simulation Applying Damage-Mechanics Model). J Solid Mech Mater Eng. $27 \quad 2013 ; 7: 102-117$. 
[3] Xie S, Liang X, Zhou H, Li J. Crashworthiness optimisation of the front-end structure of the lead car of a high-speed train. Struct Multidiscip Optim. 2016;53:339347.

[4] Shao H, Xu P, Yao S, Peng Y, Li R, Zhao S. Improved multi-body dynamics for investigating energy dissipation in train collisions based on scaling laws. Shock Vib. 2016;2016:1-11.

7 [5] Afazov S, Denmark W, Yaghi A. Modelling aspects of the design of railway 8 vehicle structures and their crashworthiness. J Rail Rapid Transit. 2015;229:1-15.

9 [6] Lu G. Energy absorption requirement for crashworthy vehicles. J Rail Rapid 10 Transit. 2002;216:31-39.

11 [7] Milho JF, Ambrósio JAC, Pereira MFOS. Design of train crash experimental tests by optimization procedures. Int J Crashworthiness. 2004;9:483-493.

[8] Dias JP, Pereira MS. Optimization methods for crashworthiness design using multibody models. Comput Struct. 2004;82:1371-1380.

[9] Milho JF, Ambrósio JAC, Pereira MFOS. A multibody methodology for the design of anti-climber devices for train crashworthiness simulation. Int $J$ Crashworthiness. 2002; 7:7-20.

18 [10] Han H-S, Koo J-S. Simulation of train crashes in three dimensions. Veh Syst 19 Dyn. 2003;40:435-450.

20 [11] Sun YQ, Cole C, Dhanasekar M, Thambiratnam DP. Modelling and analysis of the crush zone of a typical Australian passenger train. Veh Syst Dyn. 2012;50:11371155 .

23 [12] Zhou H, Zhang J, Hecht M. Three-dimensional derailment analysis of crashed freight trains. Veh Syst Dyn. 2014;52:341-361.

[13] Pawlus W, Karimi HR, Robbersmyr KG. Data-based modeling of vehicle collisions by nonlinear autoregressive model and feedforward neural network. Inf Sci. 2013;235:65-79.

[14] Fender J, Duddeck F, Zimmermann M. On the calibration of simplified vehicle crash models. Struct Multidiscip Optim. 2013;49:455-469.

[15] Taheri M, Ahmadian M. Machine learning from computer simulations with applications in rail vehicle dynamics. Veh Syst Dyn. 2016;54:653-666.

[16] Zhou H, Wang W, Hecht M. Three-dimensional override analysis of crashed railway multiple units. Veh Syst Dyn. 2012;50:663-674.

[17] Baykasoglu C, Sunbuloglu E, Bozdag SE, Aruk F, Toprak T, Mugan A. Crash and structural analyses of an aluminium railroad passenger car. Int J Crashworthiness. 2012;17:519-528.

[18] Tang Z, Liu FJ, Guo SH, Chang J, Zhang JJ. Evaluation of coupled finite element/meshfree method for a robust full-scale crashworthiness simulation of railway vehicles. Adv Mech Eng. 2016;8:1687814016642954.

[19] Li S, Zheng Z, Yu J. Energy-absorbing structure design and crashworthiness analysis of high-speed trains. Explos Shock Waves. 2015;35:164-170.

[20] Fernández-Delgado M, Cernadas E, Barro S, Amorim D. Do we need hundreds of classifiers to solve real world classification problems? J Mach Learn Res. 2014; $15: 3133-3181$.

[21] Kotsiantis S. Supervised Machine Learning: A Review of Classification Techniques. Informatica. 2007;31:249-268.

[22] Criminisi A, Shotton J. Decision forests for computer vision and medical image analysis: Springer Science \& Business Media; 2013.

[23] Louppe G. Understanding Random Forests [Ph.D.]: University of Liège; 2014. 
1 [24] Pereira M, Hecht M, Segurado V. LRV Static-passive Safety in Urban Railway 2 Systems. SAFETRAM report, December, 2000.

3 [25] Klausen A, Tørdal SS, Karimi HR, Robbersmyr KG, Jecmenica M, Melteig O. 4 Firefly optimization and mathematical modeling of a vehicle crash test based on single5 mass. J Appl Math. 2014;2014:1-10.

6 [26] Standard E. EN15227: 2008. Railway Applications-Crashworthiness 7 Requirements for Railway Vehicle Bodies, English Version, European Committee for 8 Standardization. 2010. 